\title{
A Rare Etiology of Intestinal Obstruction: Paraduodenal Hernia
}

\author{
Mustafa Yener Uzunoglu ${ }^{\mathrm{a}}$, Fatih Altintoprak ${ }^{\mathrm{b}, \mathrm{c}}$, Enis Dikicier ${ }^{\mathrm{a}}$, Ismail Zengin ${ }^{\mathrm{a}}$, \\ Yusuf Arslan ${ }^{\mathrm{a}}$
}

\begin{abstract}
Internal hernias are a rare cause of intestinal obstructions and responsible for $1 \%$ of all obstructions. Paraduodenal hernia is the most common form of congenital internal hernia and constitutes a protrusion of bowel into the retroperitoneal space through peritoneal defects near the third and fourth portion of the duodenum. The clinical symptoms are non-specific and it depends on the degree of obstruction. Preoperative diagnosis is very difficult. If diagnosis is delayed, the course of this disease may lead to high mortality and morbidity ratio.
\end{abstract}

Keywords: Internal hernia; Intestinal obstruction; Paraduodenal hernia

\section{Introduction}

Internal hernias are a rare cause of intestinal obstructions and these are responsible for $1 \%$ of all obstructions. Paraduodenal hernia is the most common form of congenital internal hernia and constitutes a protrusion of bowel into the retroperitoneal space through peritoneal defects near the third and fourth portion of the duodenum. It may lead to intestinal obstruction or is typically detected incidentally at autopsy or laparotomy. They are divided into distinct subgroups based on the localization: the paraduodenal area is the most common type with an overall incidence of $53 \%$, followed by pericecal $(13 \%)$, foramen of Winslow and transmesenteric (8\%), intersigmoid, supravesical and pelvic (6\%), and transomental (1-4\%) localizations [1-3]. There are two types of congenital paraduodenal hernia: leftsided, which is more common (75\%), and right-sided, which is

Manuscript accepted for publication April 19, 2016

aDepartment of General Surgery, Sakarya University Research and Educational Hospital, Sakarya, Turkey

bepartment of General Surgery, Sakarya University Faculty of Medicine, Sakarya, Turkey

${ }^{\mathrm{c} C}$ Corresponding Author: Fatih Altintoprak, Department of General Surgery, Sakarya University Faculty of Medicine, Sakarya, Turkey.

Email: fatihaltintoprak@yahoo.com

doi: http://dx.doi.org/10.14740/jmc2491w very rare $(25 \%)$ [4]. There is a sex predilection with a male to female ratio of $3: 1[2,5]$. The reported lifetime risk of obstruction and bowel strangulation is around $50 \%$ with a mortality of $20 \%$ and higher $[4,6]$. In this paper, we discuss about one case of left paraduodenal hernia treated successfully.

\section{Case Report}

An 18-year-old man was admitted to emergency service with the complaints of abdominal pain, nausea and vomiting. In history, he did not have any abdominal operation and he was admitted to emergency service with same complaints 1 year ago. On admission, only abdominal tenderness was detected in physical examination. Hematological and biochemical parameters were normal. The signs of intestinal obstruction were revealed in conventional abdominal graphs (Fig. 1). The dilatation of all intestine segments (Fig. 2) and minimal free fluid

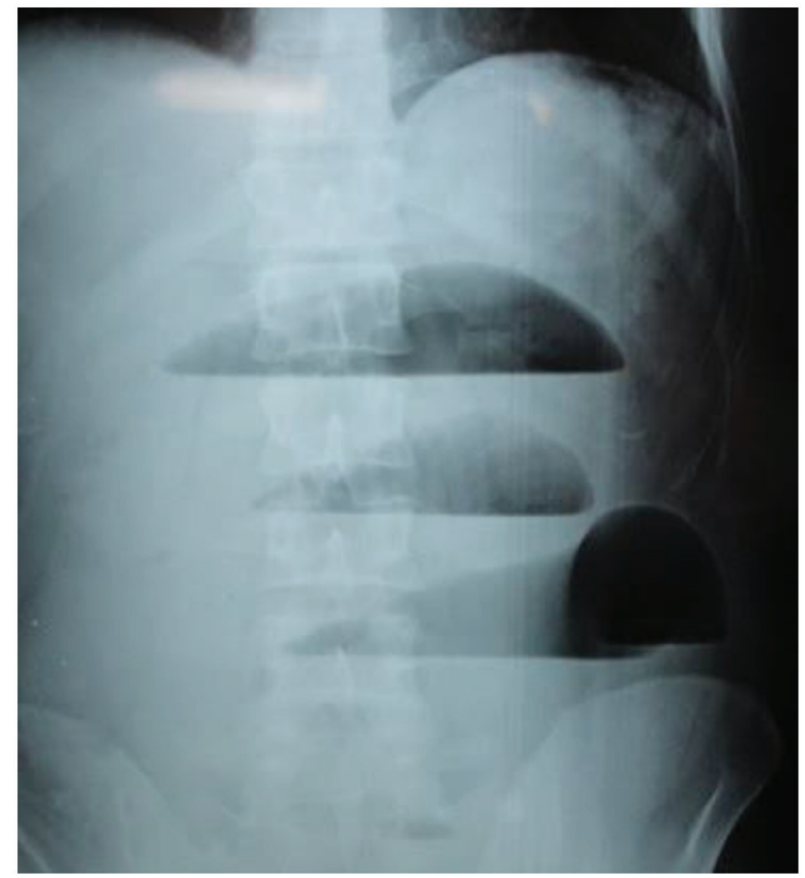

Figure 1. The signs of intestinal obstruction were revealed in conventional abdominal graphs. 


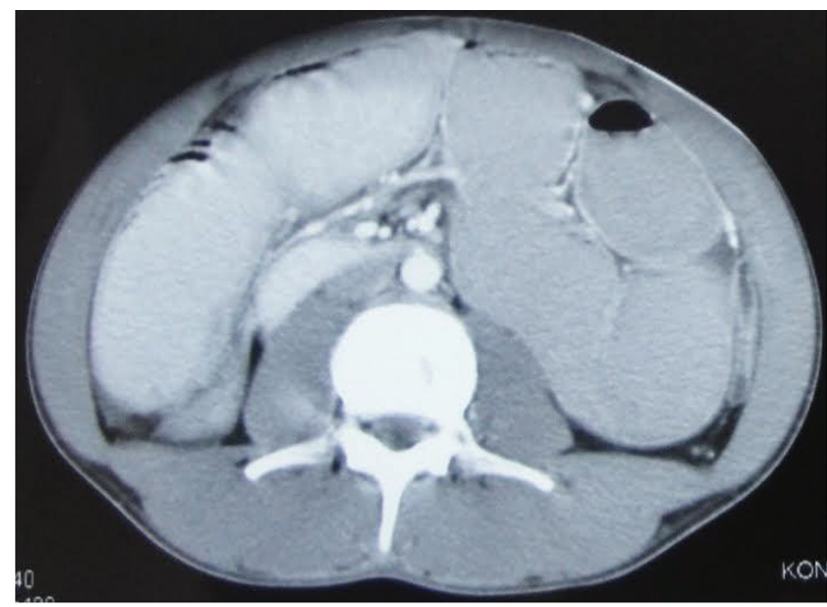

Figure 2. The dilatation of all intestine segments and minimal free fluid in pelvic area were revealed in abdominal CT.

in pelvic area were revealed in abdominal CT. In operation, herniation of the jejunal segments was detected in pouch at left side of the fourth portion of the duodenum (Fig. 3). Retracted to the abdomen of jejunal segments from pouch, detorsion and pouch was closed by non-absorbable sutures. The patient had active bowel sounds on postoperative day 2 and resumed oral intake on day 3 . He was discharged on day 4 and has been well since.

\section{Discussion}

Paraduodenal hernia was first described by Treitz in 1857 [3]. The pathophysiology is not entirely clear. One theory suggests mechanical forces of undulating intra-abdominal pressure to lead to herniation in places where the peritoneum is yet incompletely fused. Andrews first described the currently accepted mechanism of formation as a type of malrotation [5]. During the period between the fifth and 11th weeks of gestation, there is a midgut rotation and the fusion of mesentery with the posterior abdominal structures from the ligament of Treitz to the right iliac fossa $[3,5,7]$. The space into which the bowel herniates is called Landzert's fossa, and is found behind the fourth part of the duodenum. At autopsy Landzert's fossa has been found to be present in approximately $2 \%$ of the population [8].

Paraduodenal hernias usually present with symptoms of acute small bowel obstruction such as nausea, vomiting, abdominal pain and distension. Sometimes, clustering of dilated loops of small bowel may be palpable. Barium studies, CT and magnetic resonance imaging (MRI) have been used to diagnose internal hernias; the yield is highest when the patient is imaged during asymptomatic episode $[9,10]$.

Once a left paraduodenal hernia is identified, operative treatment is necessary, as patients with an LPDH have a $50 \%$ lifetime risk of developing small bowel obstruction with a 20 $50 \%$ mortality rate for acute presentations [8]. Surgical intervention included reduction of the herniated small bowel loops and closure of the hernia orifice with non-absorbable sutures or

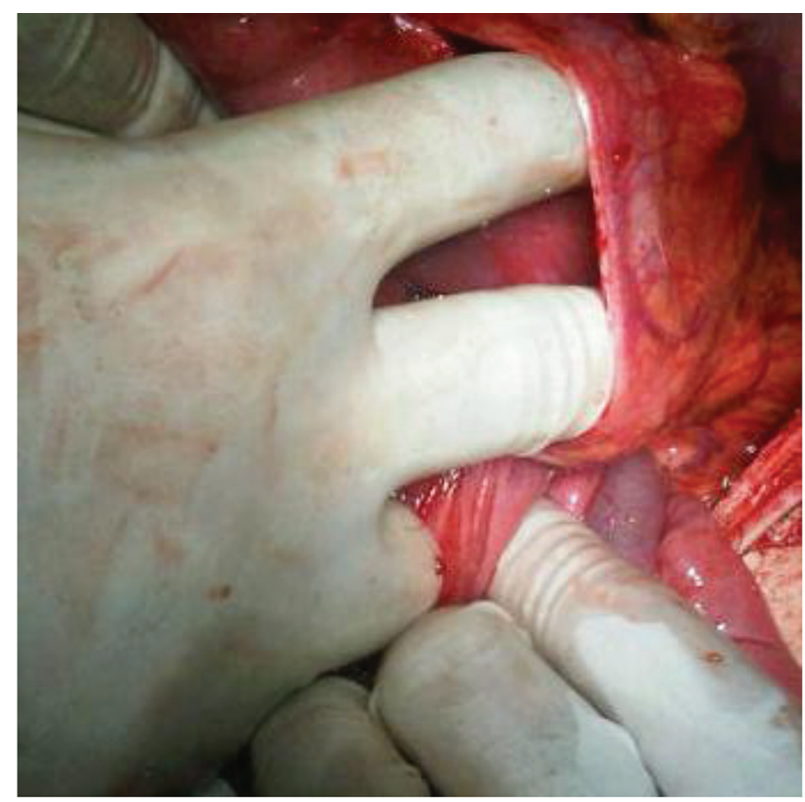

Figure 3. In operation, herniation of the jejunal segments was detected in pouch at left side of the fourth portion of the duodenum.

a mesh [6]. In our case, after reduction of the herniated small bowel loops, hernia orifice has closed with non-absorbable sutures.

In conclusion, diagnosis of this rare clinical entity is very difficult before the operation. If diagnosis is delayed, the course of this disease may be mortal due to perforation. This rare complication could be considered in young patients who have recurrent clinical symptoms of intestinal obstruction and have not any abdominal operation history. Timely surgical intervention effectively relieves the patient's complaints and prevents further complications.

\section{Conflicts of Interest}

The authors declare that they do not have any financial interest or any conflict of interest.

\section{References}

1. Husain A, Bhat S, Roy AK, Sharma V, Dubey SA, Faridi MS. Internal Hernia through Paraduodenal Recess with Acute Intestinal Obstruction: A Case Report. Indian J Surg. 2012;74(4):354-355.

2. Al-Khyatt W, Aggarwal S, Birchall J, Rowlands TE. Acute intestinal obstruction secondary to left paraduodenal hernia: a case report and literature review. World J Emerg Surg. 2013;8(1):5.

3. Akbulut S. Unusual cause of intestinal obstruction: left paraduodenal hernia. Case Rep Med. 2012;2012:529246.

4. Martin LC, Merkle EM, Thompson WM. Review of internal hernias: radiographic and clinical findings. AJR Am J Roentgenol. 2006;186(3):703-717. 
5. Manfredelli S, Andrea Z, Stefano P, Giovanni L, Maria M, Angelo F, Alberto A, et al. Rare small bowel obstruction: Right paraduodenal hernia. Case report. Int J Surg Case Rep. 2013;4(4):412-415.

6. Palanivelu C, Rangarajan M, Jategaonkar PA, Anand NV, Senthilkumar K. Laparoscopic management of paraduodenal hernias: mesh and mesh-less repairs. A report of four cases. Hernia. 2008;12(6):649-653.

7. Yun MY, Choi YM, Choi SK, Kim SJ, Ahn SI, Kim KR. Left paraduodenal hernia presenting with atypical symptoms. Yonsei Med J. 2010;51(5):787-789.
8. Amodio PM, Alberti A, Bigonzoni E, Piciollo M, Fortunati T, Alberti D. [Left paraduodenal hernia: a case report and review of the literature]. Chir Ital. 2008;60(5):721724.

9. Yoo HY, Mergelas J, Seibert DG. Paraduodenal hernia: a treatable cause of upper gastrointestinal tract symptoms. J Clin Gastroenterol. 2000;31(3):226-229.

10. Kandpal H, Sharma R, Saluja S, Sahni P, Vashisht S. Combined transmesocolic and left paraduodenal hernia: barium, CT and MRI features. Abdom Imaging. 2007;32(2):224-227. 